\title{
Comunicação entre equipes e a transferência do cuidado de pacientes críticos
}

\author{
Communication between teams and the care transfer of critical patients
}

\section{Como citar este artigo:}

Petry L, Diniz MBC. Communication between teams and the care transfer of critical patients. Rev Rene. 2020;21:e43080. D0I: https://doi. org/10.15253/2175-6783.20202143080

(DLetícia Petry ${ }^{1}$

(D)Marisa Basegio Carretta Diniz ${ }^{1}$

${ }^{1}$ Hospital de Clínicas de Passo Fundo.

Passo Fundo, RS, Brasil

\section{Autor correspondente:}

Letícia Petry

Rua Tiradentes, 295, Centro,

CEP: 99010-260. Passo Fundo, RS, Brasil.

E-mail: enfleticiapetry@outlook.com

\section{RESUMO}

Objetivo: compreender o processo de comunicação entre os profissionais de saúde durante a transferência do cuidado intra-hospitalar do paciente crítico. Métodos: estudo qualitativo, com coleta de dados por meio de entrevista com técnicos de enfermagem, enfermeiros e médicos atuantes na Unidade de Emergências Médicas e Unidade de Terapia Intensiva Adulto, perfazendo 18 profissionais. Os dados foram apreciados segundo o Discurso do Sujeito Coletivo. Resultados: evidenciou-se fragilidades no conhecimento dos profissionais quanto aos seus papeis e uma comunicação verbal estabelecida de modo superficial, como repercussão da impropriedade na utilização do instrumento de transferência existente, dificultando a obtenção de uma linha de cuidado contínua. Conclusão: a transferência do cuidado é executada entre os cenários, entretanto, o processo de comunicação se estabelece de maneira frágil e apresenta lacunas decorrentes da inexistência de um protocolo e do pouco reconhecimento acerca de sua importância por parte dos profissionais.

Descritores: Cuidados Críticos; Segurança do Paciente; Comunicação; Qualidade da Assistência à Saúde.

\footnotetext{
ABSTRACT

Objective: understanding the process of communication between professionals during intra-hospital care transfers of critical patients. Methods: qualitative study, whose data collection was carried out through an interview with nursing technicians, nurses, and physicians who worked in the Medical Emergencies Unit and the Adult Intensive Care Unit, a total of 18 professionals. Data was evaluated according to the Discourse of the Collective Subject. Results: the knowledge of professionals with regards to their role was found to have gaps, and verbal communication was superficial as a result of the misuse of the instrument of care transfers in place, which made it difficult to follow the same path of care. Conclusion: the transfer of care is carried out between settings. However, the communication process is frail and presents shortcomings resulting from the lack of a proper protocols and the little knowledge about their importance by the professionals.

Descriptors: Critical Care; Patient Safety; Communication; Quality of Health Care.
} 


\section{Introdução}

A segurança do paciente é uma temática que ganha cada vez mais espaço no meio hospitalar, cujos objetivos e ações nesse sentido são amplamente discutidose trabalhados na atualidade como meta para as instituições de saúde. $\mathrm{Na}$ busca pela qualificação dos cuidados ofertados nestes cenários, a segurança do paciente apresenta-se como aspecto indispensável à redução de erros, por meio das seis metas recomendadas pela Joint Commission International (JCI) ${ }^{(1)}$.

Diante do exposto, esta investigação direciona-se à segunda meta de segurança do paciente e tem como objeto a transferência do cuidado intra-hospitalar. Considera-se que a temática está relacionada à todas as etapas do cuidado e revela-se como facilitadora da continuidade deste entre as unidades. Desse modo, a comunicação efetiva surge como base e requer o compartilhamento de informações em um processo formado pela transferência e aceitação da responsabilidade pelos aspectos envolvidos ${ }^{(2)}$.

Reconhece-se os processos de comunicação como complexos e dinâmicos no ambiente hospitalar, caracterizado pelo alto fluxo de informações, profissionais de diferentes equipes e vasta demanda de atividades. Esses elementos geram uma necessidade permanente de atualização e troca de informações entre equipes, pacientes e familiares ${ }^{(3)}$. Nesse sentido, é preciso levar em consideração o fator humano, cuja associação está vinculada a um maior número de eventos adversos, destacando-se a falta de comunicação. Falhas encontradas na comunicação entre as unidades e entre as equipes acarretam em comprometimento da segurança do paciente, gerando quebra na continuidade do cuidado ${ }^{(2)}$.

$\mathrm{Na}$ perspectiva de qualificar o processo de comunicação, Joint Commission destaca as transferências de alta performance. Ainda, relaciona a ocorrência de eventos adversos à comunicação inefetiva e fragilidades de protocolos. A transferência da respon- sabilidade pelo cuidado é considerada ferramenta essencial para constituição da segurança, o que implica a utilização de uma comunicação efetiva ${ }^{(3)}$.

Na assistência a pacientes críticos, em razão do elevado risco de óbito existente, há a demanda de um processo consolidado pela comunicação efetiva nos diferentes cenários para prevenção da piora do quadro clínico e/ou desfecho desfavorável. A maior vulnerabilidade deve-se a gravidade das doenças e necessidade de cuidados específicos. Desta forma, a segurança do paciente precisa de uma atenção diferenciada $a^{(4)}$. Nesse meio, aponta-se que falhas de comunicação contribuem para a descontinuidade do cuidado, podendo gerar erros e eventos adversos ${ }^{(5)}$.

Há de se destacar que é essencial uma boa interação entre os diversos setores hospitalares, com vistas à promoção de uma assistência segura e de qualidade ao paciente ${ }^{(6)}$. Além disso, o trabalho em equipe é considerado impulsionador de transformações, sendo a comunicação efetiva o ponto central ${ }^{(7)}$.

Parte-se do pressuposto que a transferência do cuidado constitui-se como estratégia para o alcance do cuidado seguro, visto que diferentes profissionais e cenários compõem o percurso do paciente no ambiente intra-hospitalar. Esta investigação volta-se para a transferência do cuidado e o processo de comunicação que ocorre de um cenário para outro, mais especificamente, da Unidade de Emergências Médicas para a Unidade de Terapia Intensiva Adulto e desta para as unidades de internação.

O estudo justifica-se, portanto, na necessidade de conhecer o processo para qualificá-lo, visando sua efetividade. Nesse entendimento, traçou-se como questão norteadora de pesquisa: como ocorre o processo de comunicação entre os profissionais envolvidos na transferência do cuidado intra-hospitalar do paciente crítico e sua interferência na segurança do paciente? Na busca de tais respostas, o objetivo foi compreender o processo de comunicação entre os profissionais de saúde durante a transferência do cuidado intra-hospitalar do paciente crítico. 


\section{Métodos}

Trata-se de estudo qualitativo, realizado em um hospital de grande porte da região norte do Estado do Rio Grande do Sul. Os dados foram obtidos por meio de entrevistas com técnicos de enfermagem, enfermeiros e médicos atuantes na Unidade de Emergências Médicas e na Unidade de Terapia Intensiva Adulto, perfazendo 18 profissionais. Os sujeitos contemplam as categorias profissionais de forma paritária, com um representante de cada um dos três turnos de trabalho das unidades, escolhidos em razão da atuação direta no cuidado do paciente crítico e na transferência deste. 0 número de sujeitos foi determinado por amostra proposital ou intencional ${ }^{(8)}$. Como critério de inclusão definiu-se período de atuação superior a seis meses na instituição, delimitado com a intenção de uniformizar o tempo de experiência e evitar a possibilidade de desconhecimento quanto ao processo investigado.

Os dados foram coletados no período de abril a julho de 2019, em única etapa, por meio de entrevistas com instrumento composto por sete questões semiestruturadas elaboradas pelas pesquisadoras. As questões demandaram ao sujeito conceituar o que é transferência do cuidado e como ela se estabelece na rotina entre as unidades alvo e expressar-se acerca dos profissionais envolvidos e seus papeis, cuidados executados durante o processo, transmissão de informações e avaliação do funcionamento. As entrevistas foram realizadas em um espaço no ambiente de trabalho durante a jornada do participante, gravadas em sistema digital e transcritas na íntegra para posterior análise.

Para a apreciação dos dados, utilizou-se o Discurso do Sujeito Coletivo (DSC), método cuja técnica propõe a construção de um discurso coletivo redigido na primeira pessoa do singular e que expressa um conjunto de discursos individuais semelhantes ou complementares $^{(9)}$. Esta construção estrutura-se por meio de quatro figuras metodológicas: as expressões chave, as ideias centrais (IC), a ancoragem e, como resultado, o DSC.
Metodologicamente, foram analisados o conjunto de discursos individuais referentes a cada pergunta por meio de etapas sequenciais, culminando na construção do DSC. Foram extraídos os trechos que denotam a essência do conteúdo de cada fala, obtendo-se as expressões chave. A partir destas, foram destacadas as expressões linguísticas que exprimem de maneira sintética o tema do conjunto de expressões chave, o que resultou nas ideias centrais. Elaborou-se então o DSC relacionado a cada ideia central. Ressalta-se que não foram identificadas ancoragens pertinentes durante as análises.

A pesquisa foi conduzida de acordo com os preceitos éticos exigidos pela Resolução 466/2012 do Conselho Nacional de Saúde do Ministério da Saúde, obtendo aprovação pelo Comitê de Ética em Pesquisa sob o parecer no 3.225.830/2019 e Certificado de Apresentação para Apreciação Ética no 09259819.6.0000.5342 pela instituição cenário do estudo.

\section{Resultados}

Foram objeto da pesquisa 18 profissionais que perfazem igual proporção entre os setores investigados, para os quais identificou-se predominância do sexo feminino, sendo dez do sexo feminino e oito do sexo masculino, com média de idade de 31,94 $( \pm 7,31)$ anos. Quanto à média do tempo decorrido de formação em anos, técnicos de enfermagem apresentam 6,73 ( \pm $5,97)$, enfermeiros 7,61 $( \pm 5,96)$ e médicos 12,25 ( \pm $7,50)$. A média do período de trabalho na instituição em anos é de 2,83 $( \pm 2,11)$ para os técnicos de enfermagem, 3,33 $( \pm 1,83)$ para os enfermeiros e 7,08 $( \pm$ $5,14)$ para os médicos.

A abordagem realizada permite evidenciar que a transferência do cuidado é vivenciada pelos profissionais no cotidiano de trabalho em ambos os cenários e apresenta-se como fator determinante para a segurança do paciente. A partir dos resultados emergiram os DSC e o presente artigo apresenta 4 discursos encontrados, expostos pela IC seguida pelo DSC correspondente. 


\section{IC 1: Fragilidade da rotina da transferência do cui- dado frente aos papeis da equipe multiprofissio- nal}

O DSC 1 discorre acerca da rotina e organização da transferência do cuidado, revela alguns cuidados prévios ao deslocamento e expressa fragilidades, como manifestado abaixo. DSC 1: Na transferência do cuidado envolvem-se diretamente enfermeiro, médico e técnico de enfermagem. Percebo que o processo se inicia com contato telefônico entre os enfermeiros dos setores, com a transmissão de breves informações a respeito do estado clínico do paciente e a infusão de drogas. Para ocorrer a transporte o paciente precisa estar estável hemodinamicamente. Antes de sair do setor organizo o paciente e os dispositivos em uso, verifico a duração das drogas em infusão contínua, reviso a prescrição, as documentações e pendências, para não comprometer a segurança do paciente e poder informar ao profissional que assumirá o cuidado. Contudo, nem sempre todos os profissionais se envolvem nos cuidados antes da transferência do cuidado, há vezes que vejo apenas o enfermeiro e o técnico fazendo isso.

\section{IC 2: Comunicação verbal como instrumento para a transferência do cuidado}

Evidencia-se no DSC 2 que a comunicação verbal é utilizada para transmissão de informações entre os profissionais envolvidos. No entanto, pode-se identificar fragilidades decorrentes da não padronização. DSC 2: Na chegada, transmito as informações ao meu colega verbalmente, essa passagem acontece por categoria profissional. No entanto, nem sempre eu vejo todos passando informações, às vezes somente o médico passa. Vejo que a comunicação tem muita importância, é preciso transferir todas as informações para que o profissional possa assumir o cuidado. Entretanto, as informações ficam a critério do profissional que está transferindo, pois não temos um protocolo a ser seguido. Geralmente o médico passa a história e condição clínica, o que foi feito e o plano terapêtico, o enfermeiro passa os procedimentos realizados, o que está recebendo de medicação e as pendências e o técnico de enfermagem mais as questões de cuidados. Todavia isso não é uma regra, há desconhecimento das informações do paciente, raramente é uma conversa coletiva, às vezes os técnicos não passam nada e os médicos conversam apenas entre si.

\section{IC 3: Impropriedade na utilização de instrumento escrito para transferência do cuidado}

O DSC 3 esclarece como é utilizado o instrumento destinado à transferência do paciente, evidenciando lacunas importantes no processo. DSC 3: Para a transferência do paciente existe um instrumento que preencho com as principais informações como a história clínica, condutas empregadas, terapêtica e antibioticoterapia em uso, pendências e sinais vitais antes da saída ao setor destino. Porém, vejo diferentes profissionais preenchendo, não há uma padronização sobre a responsabilidade do preenchimento. Também percebo que há vezes o instrumento não vem com o paciente ou está incompleto.

\section{IC 4: Falhas no processo de comunicação interpon- do-se na garantia da segurança do paciente}

O DSC 4 faz referência a uma avaliação do processo de transferência do paciente e os aspectos que fragilizam o cuidado seguro no que se relaciona à comunicação efetiva. DSC 4: Acredito que haja importantes faIhas na comunicação, na maioria das vezes as informações se perdem, a equipe que vem trazer o paciente não o conhece ou não tem propriedade para passar alguma informação. As informações são insuficientes e tornam esse momento confuso e o que é transmitido depende muito dos profissionais que estão executando. Além disso, percebo um desinteresse pela transferência do cuidado, muitas vezes não sou bem recebido e não dispensam a atenção necessária para o momento.

\section{Discussão}

O estudo encontrou limitações determinadas por sua execução em apenas dois cenários de uma única instituição hospitalar. Considera-se que a transferência do cuidado do paciente crítico na maioria das vezes é iniciada com o transporte extra-hospitalar no local em que ocorreu o agravo. Assim, extrapolar as portas institucionais permitiria um olhar mais amplo sobre a importância desta transferência, à medida que perpassa diferentes profissionais e cenários, mas mantém como objetivo a construção de uma linha contínua de cuidado. Ademais, a associação do método 
utilizado com a observação do processo e uma análise documental permitiram achados mais abrangentes.

Os resultados expressos nos discursos evidenciaram que os processos de comunicação e transferência do cuidado se estabelecem de maneira frágil e são submetidos ao entendimento de cada profissional, visto que não há uma padronização para execução. As fragilidades foram vinculadas à inexistência de uma rotina pré-estabelecida e não totalmente conhecida pelos profissionais no DSC 1.

Nessa perspectiva, se reconhece que a comunicação entre equipes está permeada de dificuldades, sendo que a falta de padronização e familiarização dos profissionais com as rotinas torna as dificuldades ainda maiores e diminui o potencial das ferramentas para aprimorar a comunicação $0^{(10)}$. Assim, diante da carência de um protocolo, evidenciada pelo discurso, é preciso concordar com a predição de maior risco de ocorrência de eventos adversos na transferência de pacientes instáveis e com a necessidade de criar estratégias para barrar os erros dos profissionais, como a implantação de protocolos ${ }^{(2)}$.

Corroborando a necessidade de um protocolo para direcionar os profissionais quanto aos seus papeis durante a transferência do cuidado, destaca-se que os impactos sobre a segurança do paciente são resultados da implementação de estratégias de forma ampla e de sua utilização de forma sistematizada pelas equipes a partir de protocolos assistenciais bem definidos $^{(11)}$.

No DSC 1 foram expressos alguns cuidados realizados anteriormente à transferência, como a transmissão de informações por meio de contato telefônico, revisão dos cuidados já implementados no paciente e organização dos dispositivos e documentos, os quais foram associados à garantia da segurança do paciente. De fato, os cuidados encontrados no discurso são preconizados para a transferência, sobretudo levandose em consideração as necessidades do paciente, pois o êxito do processo advém do planejamento e da atuação organizada de toda a equipe multiprofissional ${ }^{(12)}$. Ainda que tenha sido revelada a execução de cuidados que precedem a transferência, identificou-se que a prática não é realizada por todos os profissionais envolvidos. Assim sendo, abre-se margem à existência de eventos adversos, o que sinaliza um processo que requer aprimoramento.

Sobre a análise do segundo discurso observouse também que há um reconhecimento acerca da necessidade de estabilidade hemodinâmica do paciente como critério central para conduzi-lo a outro cenário. Este achado apoia-se na assertiva de que a avaliação e estabilização clínica mínima realizadas previamente ao deslocamento propõe-se ao resguardo da segurança do paciente ${ }^{(11)}$.

No que se refere ao DSC 2, evidenciou-se reconhecimento dos profissionais quanto à importância de contemplar todas as informações relacionadas ao paciente durante a transferência para outro profissional. À vista do exposto, o estabelecimento da continuidade do cuidado demanda a transferência tanto de informações quanto da responsabilidade entre os profissionais $^{(10)}$.

Nessa sequência, o segundo discurso apontou que a transferência do cuidado nos cenários investigados é primordialmente desenvolvida valendo-se do recurso da comunicação verbal, o qual sobressai com maior utilização. Diante disso, é preciso pontuar as dificuldades existentes na comunicação entre os profissionais, o que interfere na execução do trabalho em equipe e na continuidade dos cuidados ${ }^{(11)}$. Ademais, quando analisado o transporte de pacientes críticos, estão vinculadas à ocorrência de eventos adversos situações de alterações fisiológicas, falha em equipamentos e principalmente a falta de conhecimento dos profissionais e falhas na comunicação entre a equipe que encaminha e a que recebe o paciente ${ }^{(12)}$.

Falhas de comunicação também foram sinalizadas pelos sujeitos e referem-se à fragmentação na transmissão de informações por categoria profissional, à abstenção da participação de alguns profissionais durante a transferência de informações e à relação de dependência dos dados emitidos conforme o executor, como consequência da falta de um protoco- 
lo. Contudo, sabe-se que compartilhamento de informações entre equipes desempenha importante papel para a garantia da continuidade da assistência. Para isso recomenda-se que os dados sobre o paciente sejam compartilhados de maneira completa, clara e objetiva, contemplando a emissão de todas as informações, com vistas a viabilizar o monitoramento, avaliação e planejamento do cuidado ${ }^{(13)}$.

Diante das falhas referidas na transmissão de informações, enfatiza-se a comunicação como mecanismo para integrar a equipe multiprofissional e evitar distorções e falhas no atendimento, pois através dela são fornecidas informações que contribuem para o conhecimento uniforme entre os atores do cuidado $^{(14)}$. Nesse sentido, embora esteja reconhecida a importância das estratégias de comunicação verbal, estas tornam-se mais efetivas quando seguradas por um registro escrito. A associação visa garantir a manutenção das informações indispensáveis sobre o paciente no decorrer de todas as oportunidades de transferência do cuidado ${ }^{(15)}$.

No DSC 3 constatou-se que um instrumento destinado à transferência do paciente, composto por informações relevantes para a continuidade do cuidado é utilizado na instituição. Contudo, foram reveladas fragilidades em sua utilização relacionadas a inexistência de uma padronização quanto ao profissional responsável pelo preenchimento, bem como a ocorrência da não utilização do instrumento ou de apresentar-se incompleto.

Um mapeamento realizado com documentos de transferência corrobora estes achados ao evidenciar variabilidade e inconsistência, tais documentos sendo sinalizados pela equipe receptora como incompletos e de baixa qualidade. Como causa apontada para a problemática cita-se as trocas de profissionais nos turnos de trabalho como fator influente para desconhecimento de todas as informações do paciente ${ }^{(16)}$.

Não obstante as dificuldades que permeiam a implementação de um instrumento para essa finalidade, as notas de transferência de pacientes entre setores são utilizadas como estratégia para comunicação efetiva ${ }^{(11)}$. Desse modo, propõe-se que seja empregada a maneira que mais se adeque à realidade local para deveras oportunizar a transferência de todas as informações necessárias e viabilizar a continuidade do cuidado seguro e de qualidade ${ }^{(3)}$.

Nesse sentido, a utilização do instrumento precisa ser compreendida pela equipe como ferramenta facilitadora no processo comunicativo entre os profissionais, com a finalidade de disponibilizar acesso rápido às informações e apoiar a comunicação verbal, oportunizando a continuidade do plano terapêutico nos diferentes cenários. Na direção de alcançar a utilização adequada do documento faz-se necessário aliar ações educativas ao processo ${ }^{(15)}$.

Outro extrato do DSC 3 revelou uma rotina de verificação e registro dos sinais vitais previamente ao deslocamento como um cuidado direcionado à segurança. De acordo com a literatura, os sinais vitais são informações importantes para efetividade da transferência do cuidado e embasamento das condutas clínicas, dado que insuficiências neste conteúdo são apontadas como fatores de risco para eventos adversos durante a internação $0^{(17)}$.

Na perspectiva do DSC 4 foram referidas falhas no processo de comunicação que levam à fragilização da transferência do cuidado e assim podem culminar em comprometimento da segurança. As inconsistências foram vinculadas ao desconhecimento de informações sobre o paciente por parte dos envolvidos. Achado semelhante foi encontrado em outro estudo. Desta forma, ao saber que os profissionais que transportam o paciente a outro cenário desconhecem suas características clínicas e demanda de cuidados ${ }^{(15)}$, entende-se o quão suscetível a falhas encontra-se estruturado este processo.

$\mathrm{O}$ discurso confrontou ainda aspectos como o descrédito dos profissionais, desatenção durante a troca de informações, e a transmissão das informações submetida ao conceito subjetivo daquilo que o profissional compreende ser importante. Posto isto, sinaliza-se ruídos no ambiente de comunicação como fatores que afetam negativamente o cuidado, ou seja, 
interferências no momento da transferência de conteúdo, informações emitidas de forma incompleta ou compreendidas incorretamente relacionam-se a comprometimentos da segurança do paciente ${ }^{(5)}$.

Nesse entendimento, o contexto encontrado reforça a repercussão da carência de um protocolo sistematizado para a construção de uma linha que possa sustentar efetivamente a continuidade do cuidado no cenário subsequente. 0 alcance de uma assistência mais segura passa pela implementação de ações como o cumprimento de protocolos específicos e a utilização de barreiras de segurança funcionalmente adotadas para obter efetividade nas execuções, prevenir situações de risco e eventos adversos ${ }^{(11)}$.

Outrossim, salienta-se a complexidade da execução da transferência do cuidado sobretudo por depender da comunicação efetiva como barreira para a ocorrência de eventos adversos ${ }^{(18)}$. Posto isso, sustentar investimentos contínuos para melhoria do processo de comunicação na perspectiva da transferência do cuidado permite o alinhamento da equipe multiprofissional para a garantia de uma assistência eficiente e segura ${ }^{(18)}$.

Diante das fragilidades encontradas nos discursos, a padronização dos processos dispõe-se a evitar lacunas decorrentes da decisão do profissional quanto a quais informações devem ser emitidas ${ }^{(19)}$. Reforça-se, portanto, que as ações para a garantia da prossecução do cuidado após a transferência favorecem a segurança do indivíduo, proporcionam a qualificação dos cuidados, ao passo que reduzem custos e se desvelam como estratégias eficazes para a diminuição dos eventos adversos na assistência ${ }^{(20)}$.

\section{Conclusão}

A transferência do cuidado é executada entre os cenários. No entanto, o processo de comunicação se estabelece de maneira frágil e apresenta diversas lacunas decorrentes da inexistência de um protocolo e do pouco reconhecimento acerca da importância por parte dos profissionais envolvidos. Considera-se a transferência uma importante estratégia que merece ser melhor explorada. Para tanto, propõe-se que a comunicação verbal seja guiada por um instrumento de transferência norteador aos profissionais, visando garantir a transferência das informações imprescindíveis para a continuidade do cuidado. Torna-se relevante investir na qualidade das informações transmitidas, pois na perspectiva do planejamento da assistência individualizada, a efetividade do cuidado passa pelas informações referidas.

\section{Agradecimentos}

Ao Ministério da Saúde, pela concessão de bolsa a Letícia Petry por meio da Residência Multiprofissional em Saúde com protocolo no Sistema da Comissão Nacional de Residência Multiprofissional (SisCNRMS) no $2572 / 2017$.

\section{Colaborações}

Petry L e Diniz MBC contribuíram com a concepção e projeto, análise e interpretação dos dados, redação do artigo, revisão crítica relevante do conteúdo intelectual e aprovação final da versão a ser publicada.

\section{Referências}

1. Silva ACA, Silva JF, Santos LRO, Avelino FVSD, Santos AMR, Pereira AFM. Patient safety in the hospital context: an integrative literature review. Cogitare Enferm. 2016; 21(n.esp):1-9. doi: http://dx.doi. org/10.1590/S0080-62342013000100010

2. Ministério da Saúde (BR). Agência Nacional de Vigilância Sanitária. Assistência segura: uma reflexão teórica aplicada à prática Agência Nacional de Vigilância Sanitária [Internet]. 2017 [citado 2020 jan. 13]. Disponível em: https://proqualis. net/sites/proqualis.net/files/1\%20Assist\%C3\%AAncia\%20Segura_\%20Uma\%20reflex\%C3\%A3o\%20te\%C3\%B3rica\%20aplicada \%20 \%C3\%A0\%20pr\%C3\%A1tica.pdf 
3. Silva MF, Anders JC, Rocha PK, Silva MOV, Souza S, Carneiro ES. Transfer between hospital units: implications of communication on pediatric patient safety. Rev Enferm UFPE on line [Internet]. 2017 [cited Feb 11, 2020]; 11(10):3813-20. Available from:https://pdfs.semanticscholar.org/46b6/ cfee5b77a10768d4b69bd52b8b7c903cb7a4.pdf

4. Santos GRS, Campos JF, Silva RC. Handoff communication in intensive care: links with patient safety. EscAnnaNery.2018;22(2):e20170268.doi:http:// dx.doi.org/10.1590/2177-9465-ean-2017-0268

5. Santos GRS, Barros FM, Broca PV, Silva RC. Communication noise during the nursing team handover in the intensive care unit. Texto Contexto Enferm. 2019; 28:e20180014. doi: http://dx.doi. org/10.1590/1980-265X-TCE-2018-0014

6. Minuzzi AP, Salum NC, Locks MOH, Amante LN, Matos E. Contributions of healthcare staff to promote patient safety in intensive care. Esc Anna Nery. 2016; 20(1):121-9. doi: http://dx.doi. org/10.5935/1414-8145.20160017

7. Nogueira JWS, Rodrigues MCS. Effective communication in teamwork in health: a challenge for patient safety. Cogitare Enferm. 2015; 20(3):636-40. doi: http://dx.doi.org/10.5380/ce.v20i3.40016

8. Polit DF, Beck CT. Fundamentos da pesquisa em enfermagem. Porto Alegre: Artmed; 2011.

9. Lefèvre F, Lefèvre AM. Pesquisa de representação social: um enfoque qualiquantitativo: a metodologia do discurso do sujeito coletivo. Brasília: Liber Livro; 2012.

10. Alves M, Melo CL. Handoff of care in the perspective of the nursing professionals of an emergency unit. Rev Min Enferm. 2019; 23:e-1194. doi: http:// www.dx.doi.org/10.5935/1415-2762.20190042

11. Olino L, Gonçalves AC, Strada JKR, Vieira LB, Machado MLP, Molina KL, et al. Effective communication for patient safety: transfer note and Modified Early Warning Score Rev Gaúcha Enferm. 2019; 40(esp):e20180341. doi: https://doi. org/10.1590/1983-1447.2019.20180341

12. Pires AF, Santos BN, Santos PN, Brasil VR, Luna AA. Transporte seguro de pacientes críticos. Rev Rede Cuid Saúde [Internet]. 2015 [citado 2020 Jan 13,]; 9(2):1-4. Disponível em: http://publicacoes. unigranrio.edu.br/index.php/rcs/article/ view/2531/1313
13. Bueno BRM, Moraes SS, Suzuki K, Gonçalves FAF, Barreto RASS, Gebrim CFL. Characterization of handover from the surgical center to the intensive care unit. Cogitare Enferm. 2015; 20(3):511-7. doi: http://dx.doi.org/10.5380/ce.v20i3.40274

14. Araújo Neto JD, Silva ISP, Zanin LE, Andrade AP, Moraes KM. Healthcare professionals of an Intensive Care Unit: perception of restrictive factors of the multiprofessional work. Rev Bras Promoc Saúde. 2016; 29(1):43-50. doi: http://dx.doi. org/10.5020/18061230.2016.p43

15. Hemesath MP, Kovalski AV, Echer IC, Lucena AF, Rosa NG. Effective communication on temporary transfers of inpatient care. Rev Gaúcha Enferm. 2019; 40(esp):e20180325. doi: https://doi. org/10.1590/1983-1447.2019.20180325

16. Roberts JC, Johnston-Walker L, Parker K, Townend $\mathrm{K}$, Bickley J. Improving communication of patient issues on transfer out of intensive care. BMJ Open Quality. 2018; 7:e000385. doi: https://doi. org/10.1136/bmjoq-2018-000385

17. Cross R, Considine J, Currey J. Nursing handover of vital signs at the transition of care from the emergency department to the inpatient ward: an integrative review. J Clin Nurs. 2018; 1-12. doi: https://doi.org/10.1111/jocn.14679

18. Moraes KB, Riboldi CO, Silva KS, Maschio J, Stefani LPC, Tavares JP, et al. Transfer of the care of patients with low risk of mortality in postoperative: experience report. Rev Gaúcha Enferm. 2019; 40 (esp):e20180398. doi: https://doi. org/10.1590/1983-1447.2019.20180398

19. Penna MM, Melleiro MM. Eventos adversos decorrentes de falhas de comunicação: reflexões sobre um modelo para transição do cuidado. Rev Enferm UFSM. 2018; 8(3):616-625. doi: http://dx. doi.org/10.5902/2179769225432

20. Mendes FRP, Gemito MLGP, Caldeira EC, Serra IC, Casas-Novas MV. Continuity of care from the perspective of users. Ciênc Saúde Coletiva. 2017; 22(3):841-53. doi: https://doi. org/10.1590/1413-81232017223.26292015

\section{(cc) BY}

Este é um artigo de acesso aberto distribuído sob os termos da Licença Creative Commons 